\title{
Vitamin D Levels are Associated with Painful Diabetic Peripheral Neuropathy in Chinese Patients with Type 2 Diabetes Mellitus
}

\author{
Shenglan Ding1, ${ }^{*}$, , Shuyuan Xiong1 ${ }^{1}$, , , Bo Zhou ${ }^{1}$, Laurent Irakoze ${ }^{1}$, Shimin Fu², Shuhua Deng ${ }^{3}$ \\ and Zhiping Liu ${ }^{1}$ \\ ${ }^{1}$ Department of Endocrinology, The First Affiliated Hospital of Chongqing Medical University, Chongqing, China \\ ${ }^{2}$ Department of Emergency, The First Affiliated Hospital of Chongqing Medical University; Chongqing, China
}

${ }^{3}$ Department of Respiratory, Chengdu First People's Hospital, Chengdu, China

${ }^{\#} S D$ and SX contributed equally to this work

*Corresponding author: Zhiping Liu, Department of Endocrinology, The First Affiliated Hospital of Chongqing Medical

University, Chongqing, China

\section{ARTICLE INFO}

Received: 幽 September 01, 2020

Published: 㓞 September 11, 2020

Citation: Shenglan Ding, Shuyuan Xiong Bo Zhou, Laurent Irakoze, Shimin Fu, Shuhua Deng, Zhiping Liu. Vitamin D Levels are Associated with Painful Diabetic Peripheral Neuropathy in Chinese Patients with Type 2 Diabetes Mellitus. Biomed J Sci \& Tech Res 30(2)-2020. BJSTR. MS.ID.004933.

Keywords: Vitamin D; Painful Diabetic Peripheral Neuropathy; Type 2 Diabetes Mellitus

\section{ABSTRACT}

This study was aimed to evaluate the relationship between vitamin D and painful diabetic peripheral neuropathy (painful-DPN) in Chinese patients with type 2 diabetes mellitus (T2DM). A total of 478 patients with T2DM were enrolled in this cross-sectional study, 127 of whom had painful-DPN and 351 of whom had painless diabetic peripheral neuropathy (painless-DPN). Detailed data including basic information, anthropometric measurements, laboratory examinations and neurological assessments were collected for all patients. Painful-DPN was diagnosed through neurological assessments, including neuropathy symptom score (NSS), neuropathy disability score (NDS), nerve conduction studies and the visual analogue scale. The results showed that serum vitamin D levels were significantly lower in patients with painful-DPN than patients with painlessDPN ( $\mathrm{p}=0.012)$. Pain scores, NSS and NDS were negatively associated with vitamin D levels, while motor nerve conduction velocities, motor and sensory nerve amplitudes were positively associated with vitamin $D$ levels (all $p<0.05$ ). After adjustment for age, sex, diabetes duration, smoking, diabetic kidney disease and diabetic retinopathy, diastolic blood pressure, parathyroid hormone and HbA1c, serum vitamin D levels were independently associated with painful-DPN based on logistic regression assessments $(p=0.039)$. Moreover, diabetic retinopathy and sex were independently associated with painful-DPN ( $p=0.031 ; p=0.005$, respectively). Receiver operating characteristic analysis demonstrated that serum vitamin D levels $<10.3 \mathrm{ng} / \mathrm{mL}$ were predictive of the risk of painful-DPN $(\mathrm{p}<0.05)$. These findings revealed that lower serum vitamin $\mathrm{D}$ levels were significantly associated with painful-DPN. Further studies are needed to explore the causal relationship and pathophysiology of painful-DPN.

Abbreviations: Painful-DPN: Painful Diabetic Peripheral Neuropathy; DPN: Diabetic Peripheral Neuropathy; T2DM: Type 2 Diabetes Mellitus; RCTs: Randomized Controlled Trails; painless-DPN: Painless Diabetic Peripheral Neuropathy; BMI: Body Mass Index; SBP: Systolic Blood Pressure; DBP: Diastolic Blood Pressure; HbA1c: Glycosylated Hemoglobin;UA: Uric Acid; hs-CRP: Hypersensitive C-Reactive Protein; Ca: Calcium, P: Phosphorus, Mg: Magnesium, PTH: Parathyroid Hormone, eGFR: Estimated Glomerular Filtration Rate; 25(OH)D: 25-Hydroxyvitamin D; NSS: Neuropathy Symptom Score, NDS: Neuropathy Disability Score, HRV: Heart Rate Variability; VAL: Valsalva; VAS: Visual Analogue Scale; SD: Standard Deviation; CI: Confidence Interval; OR: Odds Ratio; AUC: Area Under The Curve; NGF: Nerve Growth Factor 


\section{Introduction}

Painful diabetic peripheral neuropathy (painful-DPN) is one of the most common phenotypes of diabetic neuropathies. According to the literature, the prevalence of painful-DPN ranges from $3.3 \%$ to $65.3 \%$ in patients with diabetes [1-3]. Painful-DPN affects patients' health-related quality of life and social function and increases their health care costs [4]. Although many studies have investigated the mechanism of pain in patients with diabetic peripheral neuropathy (DPN), the specific pathophysiology of painful-DPN is not well understood [5].

Vitamin D has a well-known role in the regulation of bone metabolism and calcium homeostasis. During recent years, studies have suggested that vitamin D is associated with dozens of diseases, such as cardiovascular diseases, autoimmune diseases, cancers, and metabolic syndrome, including type 2 diabetes mellitus (T2DM) [6-8]. Many studies have further evaluated the relationship between serum vitamin D levels and DPN in patients with type 2 diabetes [9-12]. A meta-analysis including 1368 individuals with type 2 diabetes showed a significant association between vitamin D deficiency and DPN [13]. Those findings link vitamin D with DPN. Meanwhile, several observational studies have reported that patients with chronic pain have low serum vitamin D levels $[14,15]$. A number of Randomized Controlled Trails (RCTs) using vitamin D for the treatment of pain have been conducted. A meta-analysis including 19 RCTs founded that vitamin D supplementation led to a significantly greater mean decrease in pain score compared to placebo in patients with chronic pain [16].

Based on these findings, we hypothesize that serum vitamin D levels may be related to painful-DPN. However, most studies reporting the relationship between vitamin D and DPN have not assessed the differences in serum vitamin D levels between painfulDPN and painless diabetic peripheral neuropathy (painless-DPN) in patients with T2DM. There is also a lack of reports in Chinese patients. Therefore, the aim of this study was to investigate the association between serum vitamin D levels and painful-DPN in T2DM in Chongqing, China.

\section{Patients and Methods}

\section{Study Design and Patients}

This was a cross-sectional study, and patients with T2DM were consecutively recruited from the First Affiliated Hospital of Chongqing Medical University from May 2018 to January 2019. The diagnosis of T2DM was based on World Health Organization criteria. Participants $\geq 18$ years old who were using oral hypoglycemic agents and (or) insulin were included in the study. The exclusion criteria were as follows:

a) Non-diabetic neuropathies

b) Non-neuropathic pain c) Neuropathic pain due to other causes than DPN

d) Central nervous system lesions

e) Regular or high-dose vitamin $\mathrm{D}$ or calcium supplementation. This study was conducted according to the principles of the Declaration of Helsinki and was approved by the Ethics Committee of the First Affiliated Hospital of Chongqing Medical University (number 2018-008). All participants provided written informed consent.

\section{Basic Information and Anthropometric Measurements}

Patients' basic information including age, sex, body mass index (BMI), systolic and diastolic blood pressure (SBP and DBP), duration of diabetes, smoking, family history of diabetes and hypertension, and presence of diabetic retinopathy and diabetic kidney disease were collected through a questionnaire. The BMI was calculated as the weight in kilograms divided by the square of the height in meters. Current smokers or ex-smokers were defined as smokers. The diagnosis of diabetic kidney disease and diabetic retinopathy was performed according to the Guidelines for the Prevention and Treatment of Type 2 Diabetes in China [17].

\section{Laboratory Measurements}

The laboratory profile included Glycosylated Hemoglobin (HbA1c), Uric Acid (UA), Hypersensitive C-Reactive Protein (hsCRP), Calcium (Ca), Phosphorus (P), Magnesium (Mg), Parathyroid Hormone (PTH), Estimated Glomerular Filtration Rate (eGFR) and 25-hydroxyvitamin D [25(OH)D]. HbA1c was measured through high-pressure liquid chromatography (premier Hb9210, Primus, USA). UA was evaluated through an enzymatic method, and hsCRP was tested through immunoturbidimetry (Cobas c701, Roche, Germany). Ca, P and Mg levels were measured according to colorimetry (Cobas c701, Roche, Germany). PTH was detected through a chemiluminescence assay (Beckman Coulter, USA). The eGFR was calculated using the CKD-EPI equation [18]. The levels of $25(\mathrm{OH}) \mathrm{D}$ in serum or plasma indicated the vitamin D status. A LIAISON@ 25 OH Vitamin D TOTAL Assay (DiaSorin Inc, USA) was used to measure the serum levels of $25(\mathrm{OH}) \mathrm{D}$.

\section{Neurological Measurements}

The Neuropathy Symptom Score (NSS) and the Neuropathy Disability Score (NDS) have been widely used for screening of DPN in recent years [19-21]. Symptoms of DPN was assessed by the NSS, including symptoms of pain, cramps, numbness or aching in the legs, location of symptoms (feet, calves, elsewhere), timing of symptoms (present only during the day, both day and night, nocturnal exacerbation, ever awaken the patient from sleep) and ways to relieve the pain or discomfort (walking, standing, sitting or lying down). Signs of DPN were evaluated on the basis of the NDS, including an examination of pin-pricks, temperature sensation, vibration sensation with a $128-\mathrm{Hz}$ tuning fork, and ankle reflexes. 
The diagnostic criteria for DPN according to the NSS and the NDS scores were as follows:

a) Moderate or severe neuropathic signs (NDS=6-8; NDS $\geq 9$ )

b) Mild neuropathic signs (NDS=3-5) with moderate or severe neuropathic symptoms (NSS=5-6 or NSS=7-9). Of note, mild neuropathic signs or symptoms were not diagnostic criteria for DPN (NDS=3-5, NSS=3-4) [19]. DAN was diagnosed according to heart rate variability (HRV) during deep breathing ( $\leq 10$ times/min) and the valsalva (VAL) maneuver (ratio $\leq 1.10$ ).

Electrophysiological examinations were performed on each participant with a Dantec Keypoint full-function electromyographic evoked potential machine (Dantec Dynamics A/S, Denmark, Copenhagen). Nerve conduction of the tibial, peroneal, ulnar, radial, and median motor nerves, and the tibial, sural, ulnar, radial, and median sensory nerves on both sides, including nerve conduction velocity, amplitude, and latency, was assessed. All participants' limb temperatures were maintained between 330 and 350. Diagnosis of neuropathy was performed according to the corresponding reference for Chinese people [22]. If participants had previous results, electrophysiological examinations were not repeated.

\section{Pain Assessment}

Pain was evaluated with the Visual Analogue Scale (VAS) [23], a $10^{-\mathrm{cm}}$ long ruler. We assessed current pain intensity, and minimum and maximum pain intensity during the preceding 2 weeks. The participants were instructed to place a sliding gauge at a point on the ruler representing their pain experience. Patients with DPN were further divided into two groups on the basis of the VAS scores:
a) Painless-DPN: VAS $=0$
b) Painful-DPN: VAS $>0$; respectively).

\section{Statistical Analysis}

All analyses were conducted in the statistical package SPSS Version 23.0 (SPSS, IBM Corp, Armonk, NY, USA). The normality of the data was evaluated by using the Shapiro-Wilk test. Histograms, Q-Q plots, and variance homogeneity was evaluated by using Levene's test. Continuous variables with normal distribution are described as mean [Standard Deviation (SD)] and compared using independent samples t-tests. Non-normal continuous variables are presented as median and interquartile range and are compared with Mann-Whitney U tests. Categorical variables are presented as frequencies and percentages and compared with chi-square analyses. Spearman's correlation analyses were used to evaluate the relationships between vitamin D and pain scores, NSS, NDS and nerve conduction studies. Logistic regression analysis was performed to identify the effects of independent factors on painfulDPN. Candidate variables that had a p-value $<0.1$ in univariate analyses or that were considered clinically relevant were entered into a multivariable model. By considering the number of events available, we carefully chose the variables included to ensure parsimony of the final model. A receiver-operating characteristic curve was used to find the best cut-off of vitamin D for predicting the risk of painful-DPN. The threshold for statistical significance was set at $\mathrm{p}<0.05$.

\section{Results}

\section{Sample Characteristics}

A total of 478 patients with T2DM (painful-DPN: $n=127$; painless-DPN: $n=351$ ) participated in the study. Table 1 summarizes the demographic and laboratory data for each group. The patients with painful-DPN included more women $(p<0.001)$, more smokers $(p<0.001)$, and more patients with diabetic kidney disease and diabetic retinopathy $(p=0.044$ and $p=0.001$, respectively). Painful-DPN patients were also older and had a longer duration of diabetes ( $p=0.046, p=0.026$, respectively). Patients in the painless-DPN group had higher DBP and higher levels of HbA1c ( $p=0.011$, $\mathrm{p}=0.026$, respectively). Serum vitamin $\mathrm{D}$ levels in patients with painful-DPN were lower than those in patients with painless-DPN $(\mathrm{p}=0.012)$. Moreover, patients with painful-DPN had higher pain scores (VAS), NSS and NDS than patients with painless-DPN (all $\mathrm{p}<0.01$ ). The conduction velocity of peroneus motor nerve and tibialis, median sensory nerves in patients with painful-DPN was lower than those in patients with painless-DPN (all p<0.05). Meanwhile, the amplitude of tibialis and median motor nerves in patients with painful-DPN was lower than those in patients with painless-DPN (all $\mathrm{p}<0.05$ ).There was no significant difference in amplitude of all sensory nerves between groups (all p>0.05) (Table 1\&2).

Table 1: Demographic and laboratory data.

\begin{tabular}{|c|c|c|c|}
\hline Characteristic & Painful-DPN(n=127) & Painless-DPN(n=351) & $\mathbf{P}$ \\
\hline Female, n (\%) & $80(63.0)$ & $133(37.9)$ & $<0.001$ \\
\hline Age(year) & $66(59,71)$ & $62(54,71)$ & 0.046 \\
\hline $\mathrm{BMI}\left(\mathrm{kg} / \mathrm{m}^{2}\right)$ & $24.1(21.9,26.1)$ & $23.8(22.2,26.0)$ & 0.888 \\
\hline $\mathrm{SBP}(\mathrm{mmHg})$ & $136.8 \pm 18.9$ & $134.9 \pm 20.7$ & 0.330 \\
\hline $\mathrm{DBP}(\mathrm{mmHg})$ & $74.2 \pm 10.1$ & $77.0 \pm 12.4$ & 0.011 \\
\hline Diabetes duration(year) & $12(7,19)$ & $10(5,17)$ & 0.026 \\
\hline Smoker, n (\%) & $35(27.6)$ & $163(46.4)$ & $<0.001$ \\
\hline DM family history, n (\%) & $48(37.8)$ & $132(38.0)$ & $>0.999$ \\
\hline
\end{tabular}




\begin{tabular}{|c|c|c|c|}
\hline Hypertension family history, $\mathrm{n}(\%)$ & $34(26.8)$ & $74(21.2)$ & 0.217 \\
\hline Diabetic kidney disease, $\mathrm{n}(\%)$ & $54(42.5)$ & $114(32.6)$ & 0.044 \\
\hline Diabetic retinopathy, $\mathrm{n}(\%)$ & $63(49.6)$ & $9.3(7.5,11.4)$ & 0.001 \\
\hline HbA1c (\%) & $8.8(7.0,10.3)$ & $312(255,378)$ & 0.026 \\
\hline $\mathrm{UA}(\mu \mathrm{mol} / \mathrm{L})$ & $312(243,385)$ & $1.52(0.53,5.19)$ & 0.546 \\
\hline $\mathrm{hs}-\mathrm{CRP}(\mathrm{mg} / \mathrm{L})$ & $1.24(0.49,4.09)$ & $2.32(2.23,2.39)$ & 0.564 \\
\hline $\mathrm{Ca}(\mathrm{mmol} / \mathrm{L})$ & $2.30(2.23,2.38)$ & $1.19(1.05,1.35)$ & 0.750 \\
\hline $\mathrm{P}(\mathrm{mmol} / \mathrm{L})$ & $1.20(1.10,1.34)$ & $0.79(0.73,0.85)$ & 0.515 \\
\hline $\mathrm{Mg}(\mathrm{mmol} / \mathrm{L})$ & $0.80(0.72,0.85)$ & $36.6(25.9,49.7)$ & 0.565 \\
\hline $\mathrm{PTH}(\mathrm{pg} / \mathrm{mL})$ & $34.2(23.7,47.2)$ & $75.7(50.7,97.5)$ & 0.805 \\
\hline $\mathrm{eGFR}\left(\mathrm{mL} / \mathrm{min} / 1.73 \mathrm{~m}^{2}\right)$ & $82.2(50.7,99.0)$ & $20.7(14.7,26.4)$ & 0.012 \\
\hline
\end{tabular}

\section{The association of serum vitamin D levels with}

\section{Neuropathy parameters}

Spearman's correlation analysis showed that the pain scores (VAS), NSS and NDS were negatively associated with serum vitamin D levels $(\mathrm{p}<0.05)$. There was no significant correlation between serum vitamin D levels and DAN and HRV during deep breathing ( $p=0.858 ; p=0.379$, respectively). VAL showed a positive correlation with serum vitamin D levels $(p=0.008)$. There was a significant positive correlation between serum vitamin D levels and conduction velocity of the tibial, peroneal, ulnar, and median motor nerves $(\mathrm{p}<0.05)$. All sensory nerve conduction velocities showed no significant correlation with serum vitamin D levels (all $\mathrm{p}>0.05$ ) (Table 2). There is also a significant positive correlation between serum vitamin D levels and amplitude of tibialis, peroneus, ulnar and median motor nerves and amplitude of tibialis, ulnar sensory nerves $(\mathrm{p}<0.05)$ (Table 3).

Table 2: Spearman's correlation analysis between vitamin D and neuropathy parameters.

\begin{tabular}{|c|c|c|}
\hline Characteristic & $\mathbf{r}$ & $\mathbf{p}$ \\
\hline VAS score & -0.114 & 0.013 \\
\hline NSS & -0.120 & 0.018 \\
\hline NDS & -0.145 & 0.004 \\
\hline Autonomic neuropathy (\%) & 0.056 & 0.135 \\
\hline HRV & 0.064 & 0.379 \\
\hline Results of VAL & 0.191 & 0.008 \\
\hline Motor nerve conduction(m/s) & & 0.863 \\
\hline Right tibialis & 0.011 & 0.030 \\
\hline Left tibialis & 0.138 & 0.001 \\
\hline Right peroneus & 0.211 & $<0.001$ \\
\hline Left peroneus & 0.226 & 0.905 \\
\hline Right ulnar & 0.010 & 0.006 \\
\hline Left ulnar & 0.232 & 0.580 \\
\hline Right radial & 0.046 & 0.570 \\
\hline Left radial & 0.048 & 0.184 \\
\hline Right median & 0.117 & \\
\hline Left median & 0.179 & \\
\hline
\end{tabular}

\section{The Relationship between Vitamin D and Painful-DPN}

The effects of ten independent variables (age, sex, diabetes duration, smoking, diabetic kidney disease and diabetic retinopathy, DBP, PTH, HbA1c and vitamin D) on painful-DPN were explored by logistic regression analysis. The full model was statistically significant $(\mathrm{P}<0.001)$. As shown in Table 3 , the serum vitamin D levels were significantly associated with painful-DPN [p=0.039, OR=0.964, 95\% confidence interval (CI): 0.932-0.998]. For every $1 \mathrm{ng} / \mathrm{mL}$ increase in serum vitamin $\mathrm{D}$ level, the odds ratio (OR) of painful-DPN was reduced by $3.60 \%$. Furthermore, diabetic retinopathy and sex were independently associated with painful-DPN ( $\mathrm{p}=0.031,0 \mathrm{R}=1.813,95 \% \mathrm{CI}$ : 1.057-3.110; $\mathrm{p}=0.005$, $\mathrm{OR}=2.211,95 \% \mathrm{CI}$ : 1.277-3.830; respectively). In receiver-operating characteristic curve analysis, the Area Under the Curve (AUC) was 0.674 (95\%CI: 0.620 to $0.728, \mathrm{p}<0.001$ ). The optimal cut-off of vitamin $\mathrm{D}$ was $10.3 \mathrm{ng} / \mathrm{mL}$, and the corresponding Youden index was 0.26 (sensitivity: 78.0\%; specificity: $48.0 \%$ ) (Figure 1).

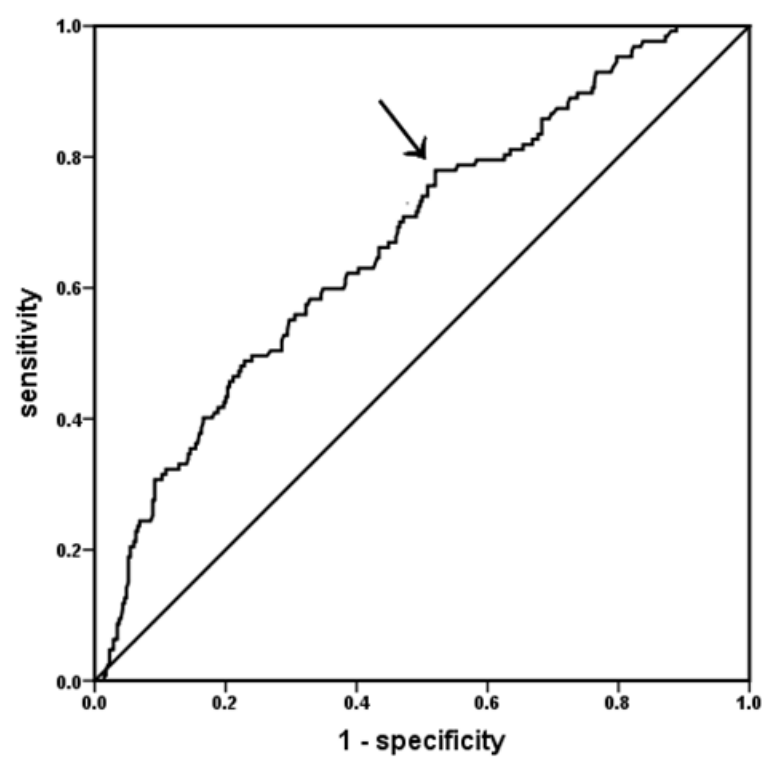

Figure 1: Receiver-operating characteristic curve analysis for predicting the risk of painful-DPN. 
Table 3: Logistic regression for related factors of painful-DPN.

\begin{tabular}{|c|c|c|}
\hline Parameter & OR(95\% CI) & p \\
\hline vitamin D & $0.964(0.932-0.998)$ & 0.039 \\
\hline${ }^{a}$ Gender & $2.211(1.277-3.830)$ & 0.005 \\
\hline${ }^{b}$ Diabetic retinopathy & $1.813(1.057-3.110)$ & 0.031 \\
\hline
\end{tabular}

\section{Discussion}

This study demonstrated that patients with painful-DPN had lower serum vitamin D levels than patients with painless-DPN. Pain scores and neuropathy parameters were significantly associated with vitamin D levels. Serum vitamin D levels were independently associated with painful-DPN in patients with T2DM. Moreover, diabetic retinopathy and sex were independently related to painfulDPN. A serum vitamin D level $<10.3 \mathrm{ng} / \mathrm{mL}$ was a significant predictor of the risk of painful-DPN. Previous studies have found that lower vitamin D levels are prevalent in patients with T2DM, especially in those with DPN [9-11,24]. Shillo et al. have reported that serum vitamin D levels were lower in patients with painful-DPN than in those with painless-DPN, and pain scores were negatively correlated with serum vitamin D levels [25]. These findings are in line with our results. However, the finding of Alkhatatbeh et al. was inconsistent with above findings [26]. Meanwhile, the relationship between serum vitamin $D$ levels and neuropathy parameters in type 2 diabetics with painful-DPN is also controversial. In patients with DPN, lower vitamin D levels have been reported to correlate with NDS and nerve conduction studies. For every $1 \mathrm{ng} / \mathrm{mL}$ increase in vitamin D, there was $2.2 \%$ and $3.4 \%$ lower risk of the presence and severity of NCV impairment, respectively $[27,28]$.

In patients with painful-DPN, Shillo et al. did not find a significant association between nerve conduction studies, DAN, and serum vitamin D levels, although HRV during deep breathing was significantly correlated with serum vitamin D levels [25]. However, our results showed that serum vitamin D levels were significantly associated with VAL, motor nerve conduction velocity and motor and sensory nerve amplitude, but not with DAN and sensory nerve conduction velocity. In addition, NSS and NDS showed a negative correlation with serum vitamin D levels in our study. The discrepancies between the results of our study and previous studies may be due to the different races and sample sizes. Furthermore, a research letter and an interventional study (without control group) have reported that vitamin D supplementation can relieve neuropathic pain in patients with T2DM, and vitamin D may play a role in the treatment of painful-DPN [29,30]. In addition, a recent observational study found that lower vitamin D levels were significantly associated with painful-DPN, compared to painlessDPN, no-DPN, and healthy volunteers [25]

The findings of our study are consistent with those results. Consequently, vitamin D may play a role in the pathophysiology of painful-DPN. Some possible mechanisms are as follows. Nerve growth factor (NGF) is well known to be beneficial for neurons
[31,32]. A vitamin D3 derivative (CB1093) has been found to increase NGF concentrations in diabetic rats [33], and active Vitamin D3 (tacalcitol) has been shown to promote expression of NGF [34]. After correction of the vitamin D deficiency, NGF production increases. Therefore, vitamin D3 may be useful for preventing and treating neurotrophic deficits. Importantly, vitamin D3 (cholecalciferol) supplementation has been found to relieve neuropathic pain in rats by inducing the dysregulation of multiple genes, an effect associated with modulating opioid signaling [35]. Interestingly, another cross-sectional study using the PainDETECT questionnaire to assess neuropathic pain showed that serum vitamin D was not associated with neuropathic pain in patients with T2DM [26]. The inconsistency may be due to the different races, sample sizes and different diagnosis criteria of painful-DPN.

Diabetic retinopathy was independently associated with painful-DPN in the present study. A previous study in the Middle East has demonstrated that diabetic retinopathy was significantly related to painful-DPN, using the Douleur Neuropathique-4 [36]. Microangiopathy is an important basis of DPN [37]. Diabetic retinopathy is one of the most common microangiopathies in diabetes mellitus. These findings suggest that microangiopathy may also play a role in the development of painful-DPN. In agreement with our results, sex was independently associated with painfulDPN in several studies [2,26,36,38,39]. Females experienced a higher frequency of neuropathic pain among diabetic patients, possibly because they have higher sensitivity and exhibit protective effects in multiple sensory domains [40]. Therefore, sex should also be considered in the detection and treatment of painful-DPN. He et al. reported the cut-off value of vitamin D for predicting the occurrence of DPN to be $16.01 \mathrm{ng} / \mathrm{mL}$ [11]. However, our study found a lower cut-off value of serum vitamin D for predicting the risk of painful-DPN $(10.3 \mathrm{ng} / \mathrm{mL})$. Notably, the cut-off value of vitamin D did not have strong sensitivity or specificity in screening for painful-DPN. This result may be because painful-DPN is a multifactorial disease, and vitamin D levels are susceptible to other factors, such as diet and sunlight exposure.

Our study demonstrated that serum vitamin D levels were significantly associated with painful-DPN, a finding that may support future exploration of the mechanisms of painful-DPN and provide a new direction for the prevention and treatment of painful-DPN. However, there are several limitations to this study. First, this study was a lack of more sensitive measures for small fiber neuropathy (than HRV and VAL) such as skin biopsy. Due to the lack of translated and sinicized neuropathic pain questionnaires in China, no neuropathic pain questionnaires were used to screen for painful-DPN in this study. Moreover, because of the cross-sectional design, a conclusion of cause and effect between the variables and painful-DPN cannot be inferred. In addition, the cutoff value of vitamin D in predicting painful-DPN could not be confirmed. Furthermore, we did not assess the effects of confounding factors, 
including sunlight exposure and diet on serum vitamin D levels. Finally, this was also a single-center study, and more studies are necessary to verify our findings.

\section{Conclusion}

In this cross-sectional study, we found that higher serum vitamin D levels may play a protective role in painful-DPN in Chinese individuals with type 2 diabetes. Serum vitamin D levels $<10.3 \mathrm{ng} / \mathrm{mL}$ may predict the risk of painful-DPN. Further large studies are needed to verify our findings and explore the underlying mechanisms.

\section{Acknowledgment}

We specifically thank all of the participants in our study.

\section{Conflict of Interest}

The authors have no conflicts of interest to disclose.

\section{References}

1. Sorensen L, Molyneaux L, Yue DK (2002) Insensate versus painful diabetic neuropathy: the effects of height, gender, ethnicity and glycaemic control. Diabetes Res Clin Pract 57(1): 0-51.

2. Jane SW, Lin MS, Chiu WN, Beaton RD, Chen MY (2016) Prevalence, discomfort, and self-relief behaviours of painful diabetic neuropathy in Taiwan: a cross-sectional study. BMJ Open 6(10): e011897.

3. Halawa MR, Karawagh A, Zeidan A, Mahmoud AEDH, Sakr M, et al (2010) Prevalence of painful diabetic peripheral neuropathy among patients suffering from diabetes mellitus in Saudi Arabia. Curr Med Res Opin 26(2): 337-343.

4. Sadosky A, Schaefer C, Mann R, Bergstrom F, Baik R, et al. (2013) Burden of illness associated with painful diabetic peripheral neuropathy among adults seeking treatment in the US: results from a retrospective chart review and cross-sectional survey. Diabetes Metab Syndr Obes 6: 79-92.

5. Tavakoli M, Mojaddidi M, Fadavi H, Malik RA (2008) Pathophysiology and treatment of painful diabetic neuropathy. Curr Pain Headache Rep 12(3): 192-197.

6. Makariou S, Liberopoulos EN, Elisaf M, Challa A (2011) Novel roles of vitamin D in disease: What is new in 2011? Eur J Intern Med 22(4): 355362.

7. Palomer X, González Clemente JM, Blanco Vaca F, Mauricio D (2008) Role of vitamin D in the pathogenesis of type 2 diabetes mellitus. Diabetes obes metab 10(3): 185-197.

8. Pilz S, Kienreich K, Rutters F, de Jongh R, van Ballegooijen AJ, et al. (2013) Role of Vitamin D in the development of insulin resistance and type 2 diabetes. Curr Diab Rep 13(2): 261-270.

9. Soderstrom LH, Johnson SP, Diaz VA, Mainous III AG (2012) Association between vitamin $D$ and diabetic neuropathy in a nationally representative sample: results from 2001-2004 NHANES. Diabet Med 29(1): 50-55.

10. Celikbilek A, Gocmen AY, Tanik N, Borekci E, Adam M, et al. (2015) Decreased serum vitamin D levels are associated with diabetic peripheral neuropathy in a rural area of Turkey. Acta Neurol Belg 115(1): 47-52.

11. He R, Hu Y, Zeng H, Zhao J, Zhao J, et al. (2017) Vitamin D deficiency increases the risk of peripheral neuropathy in Chinese patients with type 2 diabetes. Diabetes Metab Res Rev 33(2): e2820.

12. Zampelis T, Papadakis G, Kokotis P, Villiotou V, Dogkas N, et al. (2017) Lack of definite association of vitamin D deficiency with diabetic neuropathy. Investigation in Greek and in Bangladeshi patients. In vivo 31(2): 259-261.
13. Qu GB, Wang LL, Tang X, Wu W, Sun YH (2017) The association between vitamin $D$ level and diabetic peripheral neuropathy in patients with type 2 diabetes mellitus: an update systematic review and meta-analysis. J Clin Transl Endocrinol 9: 25-31.

14. Atherton K, Berry DJ, Parsons T, Macfarlane G, Power C, et al. (2009) Vitamin D and chronic widespread pain in a white middle-aged British population: evidence from a cross-sectional population survey. Ann Rheum Dis 68(6): 817-822.

15. Madani M, Masoudi AN, Taghizadeh M (2014) Non-Specific Musculoskeletal Pain and Vitamin D Deficiency in Female Nurses in Kashan, Iran. J Musculoskelet Pain 22(3): 268-274.

16. Wu Z, Malihi Z, Stewart AW, Lawes CM, Scragg R (2016) Effect of Vitamin D Supplementation on Pain: A Systematic Review and Meta-analysis. Pain Physician 19(7): 415-427.

17. Chinese Diabetes Society (2018) Guidelines for the prevention and control of type 2 diabetes in China (2017 Edition).Chin J Diabetes Mellitus 10: 4-67.

18. Levey AS, Stevens LA, Schmid CH, Zhang Y, Castro III AF, et al. (2009) A new equation to estimate glomerular filtration rate. Ann Intern Med 150(9): 604-612.

19. Young MJ, Boulton AJM, Macleod AF, Williams DRR, Sonksen PH (1993) A multicentre study of the prevalence of diabetic peripheral neuropathy in the United Kingdom hospital clinic population. Diabetologia 36(2): 150-154.

20. Daousi C, Benbow SJ, Woodward A, MacFarlane IA (2006) The natural history of chronic painful peripheral neuropathy in a community diabetes population. Diabet Med 23(9): 1021-1024.

21. Daousi C, Macfarlane IA, Woodward A, Nurmikko TJ, Bundred PE, et al. (2004) Chronic painful peripheral neuropathy in an urban community: A controlled comparison of people with and without diabetes. Diabet Med 21(9): 976-982.

22. Tang X (1995) Clinical Eceltromyography Study. The Beijing University of Science and Technology China harmony Medical college unites the publishing house: Beijing

23. Wewers ME, Lowe NK (1990) A critical review of visual analogue scales in the measurement of clinical phenomena. Res Nurs Health 13(4): 227236.

24. Kostoglou Athanassiou I, Athanassiou P, Gkountouvas A, Kaldrymides P (2013) Vitamin D and glycemic control in diabetes mellitus type 2.Ther Adv Endocrinol Metab 4(4): 122-128.

25. Shillo P, Selvarajah D, Greig M, Gandhi R, Rao G, et al. (2019) Reduced vitamin $D$ levels in painful diabetic peripheral neuropathy. Diabet Med 36(1): 44-51.

26. Alkhatatbeh M, Abdul Razzak KK (2019) Neuropathic pain is not associated with serum vitamin D but is associated with female gender in patients with type 2 diabetes mellitus. BMJ Open Diabetes Res Care $7(1): \mathrm{e} 000690$.

27. Abdelsadek SE, Saghier EOEI, Raheem SIA (2018) Serum 25(OH) Vitamin D level and its relation to diabetic peripheral neuropathy in Egyptian patients with type 2 diabetes mellitus. Egypt J Neurol Psychiatr Neurosurg 54(1): 36

28. Alamdari A, Mozafari R, Tafakhori A, Faghihi Kashani S, Hafezi Nejad $\mathrm{N}$, et al. (2015) An inverse association between serum vitamin D levels with the presence and severity of impaired nerve conduction velocity and large fiber peripheral neuropathy in diabetic subjects. Neurol Sci 36(7): 1121-1126.

29. Lee P, Chen R (2008) Vitamin D as an analgesic for patients with type 2 diabetes and neuropathic pain. Arch Intern Med (JAMA Intern Med) 168(7): 771-772.

30. Basit A, Basit KA, Fawwad A, Shaheen F, Fatima N, et al. (2016) Vitamin $\mathrm{D}$ for the treatment of painful diabetic neuropathy. BMJ Open Diabetes Res Care 4(1): e000148. 
31. Lindsay RM, Harmar AJ (1989) Nerve growth factor regulates expression of neuropeptide genes in adult sensory neurons. Nature 337(6205): 362-364.

32. Anand P, Terenghi G, Warner G, Kopelman P, Williams Chestnut RE, et al. (1996) The role of endogenous nerve growth factor in human diabetic neuropathy. Nat Med 2(6): 703-707.

33. Riaz S, Malcangio M, Miller M, Tomlinson DR (1999) A vitamin D3 derivative (CB1093) induces nerve growth factor and prevents neurotrophic deficits in streptozotocin-diabetic rats. Diabetologia 42(11): 1308-1313.

34. Fukuoka M, Sakurai K, Ohta T, Kiyoki M, Katayama I (2001) Tacalcitol, an active vitamin D3, induces Nerve growth factor production in human epidermal keratinocytes. Skin Pharmacol Appl Skin Physiol 14(4): 226233.

35. Poisbeau P, Aouad M, Gazzo G, Lacaud A, Kemme V, et al. (2019) Cholecalciferol (Vitamin D3) reduces rat neuropathic pain by modulating opioid signaling. Mol Neurobiol 56(10): 7208-7221.

\section{ISSN: 2574-1241}

DOI: $10.26717 /$ BJSTR.2020.30.004933

Shenglan Ding, Shuyuan Xiong. Biomed J Sci \& Tech Res

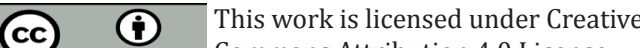

Submission Link: https://biomedres.us/submit-manuscript.php
36. Jambart S, Ammache Z, Haddad F, Younes A, Hassoun A, et al. (2011) Prevalence of painful diabetic peripheral neuropathy among patients with diabetes mellitus in the Middle East Region. J Int Med Res 39(2): 366-377.

37.Zochodne DW (2007) Diabetes mellitus and the peripheral nervous system: Manifestations and mechanisms. Muscle Nerve 36(2): 144-166.

38. Raputova J, Srotova I, Vlckova E, Sommer C, Üçeyler N, et al. (2017) Sensory phenotype and risk factors for painful diabetic neuropathy: A cross-sectional observational study. Pain 158(12): 2340-2353.

39. Cardinez N, Lovblom LE, Bai JW, Lewis E, Abraham A, et al. (2018) Sex differences in neuropathic pain in longstanding diabetes: Results from the Canadian Study of Longevity in Type 1 Diabetes. J Diabetes Complications 32(7): 660-664.

40. Hashmi JA, Davis KD (2014) Deconstructing sex differences in pain sensitivity. Pain 155(1): 10-13.

BIOMEDICAL
RESEARCHES $\quad \begin{aligned} & \text { Assets of Publishing with us } \\ & \text { - Global archiving of articles }\end{aligned}$

